05

\title{
Импульсное перемагничивание антиферромагнитных наночастиц ферригидрита
}

\author{
() Д.А. Балаев ${ }^{1,2}$, А.А. Красиков ${ }^{1}$, Д.А. Великанов ${ }^{1}$, С.И. Попков ${ }^{1,2}$, Н.В. Дубынин ${ }^{3}$, \\ С.В. Столяр ${ }^{1,2,4}$, В.П. Ладыгина ${ }^{4}$, Р.Н. Ярославцев ${ }^{1,2}$ \\ ${ }^{1}$ Институт фризики им. Л.В. Киренского, ФИЦ КНЦ СО РАН, \\ Красноярск, Россия \\ ${ }^{2}$ Сибирский федеральный университет, \\ Красноярск, Россия \\ ${ }^{3}$ Национальный исследовательский московский государственный строительный университет, \\ Москва, Россия \\ ${ }^{4}$ Президиум Красноярского научного центра, ФИЦ КНЦ СО РАН, \\ Красноярск, Россия \\ E-mail: dabalaev@iph.krasn.ru
}

(Поступила в Редакцию 13 марта 2018 г.)

\begin{abstract}
Методом импульсного магнитометра в максимальных полях $H_{\max }$ до $130 \mathrm{kOe}$ длительностью 4,8 и $16 \mathrm{~ms}$ исследованы процессы динамического перемагничивания наночастиц ферригидрита. Ферригидрит проявляет антиферромагнитное упорядочение, а нескомпенсированный магнитный момент в наночастицах возникает благодаря дефектам, что приводит к поведению, типичному для магнитных наночастиц. Измерения динамических петель гистерезиса при указанных условиях показали, что применение импульсных полей существенно расширяет температурный диапазон существования магнитного гистерезиса, а варьирование максимального поля и длительности импульса позволяет „управлять“ такой характеристикой, как коэрцитивная сила. Такое поведение вызвано как релаксационными эффектами, присущими „обычным“ ферро- и ферримагнитным наночастицам, так и особенностями, присущими антиферромагнитным наночастицам.
\end{abstract}

Исследование выполнено при финансовой поддержке Российского фонда фундаментальных исследований, Правительства Красноярского края, Красноярского краевого фонда поддержки научной и научно-технической деятельности в рамках научного проекта № 17-42-240138 „Исследование магнитных наночастиц в сильных импульсных магнитных полях“.

DOI: $10.21883 /$ FTT.2018.10.46520.062

\section{1. Введение}

Наночастицы материалов, проявляющих антиферромагнитное (AF) упорядочение, обладают интересными магнитными свойствами [1-3]. Это вызвано поверхностными эффектами и дефектами (нарушениями AF-порядка). Дефекты возникают на поверхности частиц, а также могут (в силу кристаллохимических свойств) присутствовать и в объеме частиц. Наличие дефектов приводит к тому, что в AF-наночастицах появляется нескомпенсированный магнитный момент, причем, его величина может достигать сотен магнетонов Бора при размерах несколько нанометров. Это открывает перспективы применения AF-наночастиц в различных областях, например, медицинских приложениях $[4,5]$.

Помимо существования нескомпенсированного магнитного момента $\mu_{p}$, в магнитном поведении AF-наночастиц есть еще ряд интересных эффектов. Подобно ферро- и ферримагнитным наночастицам АF-частицы также демонстрируют суперпарамагнитное (SP) поведение в области температур выше характерной температуры блокировки $T_{B}$. В области температур $T<T_{B}$ кривые намагничивания $M(H)$ демонстрируют гистерезис. С одной стороны, подобно ферро- и ферримагнитным наночастицам, гистерезис $M(H)$ связан с конкуренцией зеемановской энергии $\mu_{p} H$ и энергии магнитной анизотропии $K_{\mathrm{eff}} V$ ( $K_{\mathrm{eff}}$ - эффективная константа магнитной анизотропии, включающая и поверхностную анизотропию, $V$ - объем частицы). С другой стороны, магнитный момент АF-частички может быть обменно связан с AF-,,ядром“, и это может быть источником дополнительной анизотропии. Указанный механизм, возможно, проявляется в смещении петли магнитного гистерезиса AF-частиц после их охлаждения во внешнем поле от температуры, превышающей $T_{B}$ [6-13]. Отметим также, что кривая намагничивания ансамбля AF-наночастиц в первом приближении (без учета обменной связи $\mu_{p}$ и $\mathrm{AF}-$, ,ядра“") является суперпозицией вкладов от магнитных моментов частиц и характерного, линейного по полю, магнитного отклика от АF-упорядоченного „ядра“ $[1,2,14-21]$.

При стандартных исследованиях магнитного гистерезиса в квазистатических магнитных полях скорость изменения внешнего поля $d H / d t$ обычно не превышает $\sim 10^{2} \mathrm{Oe} / \mathrm{s}$. Однако исследование процессов динамического перемагничивания (с существенно бо́льшими значениями $d H / d t$ ) может дать новую информацию, поскольку варьирование параметра $d H / d t$ затрагивает 
релаксационные процессы [22-24]. Соотношение между значениями $T_{B}, V, K_{\text {eff }}$ и характерными временами измерения $\tau_{m}$ и релаксации частицы $\tau_{0}$, определяется выражением Нееля-Брауна (Nèel-Brown)

$$
T_{B}=K_{\mathrm{eff}} V / \ln \left(\tau_{m} / \tau_{0}\right) k_{B},
$$

$\left(k_{B}\right.$ - константа Больцмана). Величина $\tau_{0}$, может находиться в пределах $10^{-9}-10^{-12} \mathrm{~s}$, значение $\tau_{m}$ для квазистатических измерений составляет $\sim 10^{1}-10^{2} \mathrm{~s}[1]$. Увеличение параметра $d H / d t$ при намагничивании аналогично уменьшению характерного времени $\tau_{m}$, что в результате приводит к эффективному росту SP-температуры блокировки и в результате зависимость $M(H)$, обратимая в квазистационарных измерениях, может демонстрировать гистерезис в процессах динамического перемагничивания.

Если значение $H_{R}$ - поля начала обратимого поведения зависимости $M(H)$ (иными словами, при $H \geq H_{R}$ петля гистерезиса закрытая) достаточно небольшое (например, порядка $10^{3} \mathrm{Oe}$ ), то указанные процессы можно наблюдать на установках, генерирующих переменные магнитные поля различной частоты. Однако, если величина $H_{R}$ будет составлять $\sim 10^{4} \mathrm{Oe}$ и более, то процессы динамического перемагничивания возможно исследовать с помощью установок, генерирующих импульсное магнитное поле. AF-наночастицы, как раз, и относятся к классу объектов с большими значениями $H_{R}[25,17,13,26]$. Поведение такого параметра, как коэрцитивная сила $H_{C}$, возможно исследовать только с помощью магнетометров, работающих в импульсных магнитных полях большой напряженности.

Ранее в импульсных магнитных полях были исследованы процессы динамического перемагничивания наночастиц $\varepsilon-\mathrm{Fe}_{2} \mathrm{O}_{3}$ [27] (для этих объектов значение $H_{R}$ составляет $\sim 50 \mathrm{kOe})$. Из петель динамического магнитного гистерезиса были получены зависимости коэрцитивной силы как функции скорости изменения внешнего поля $d H / d t$, что позволило на основании теоретической модели для ферромагнитных наночастиц [22,23] выявить роль поверхностной анизотропии для указанных объектов. По нашему мнению, логично распространить подобные исследования процессов динамического перемагничивания на AF-наночастицы. Целью данной работы является установление закономерностей в поведении петель динамического магнитного гистерезиса (ДМГ) антиферромагнитно упорядоченного ферригидрита.

Ферригидрит является гидрооксидом железа (номинальная формула $\left.5 \mathrm{Fe}_{2} \mathrm{O}_{3} \cdot 9 \mathrm{H}_{2} \mathrm{O}\right)$ и существует в наноразмерном виде. Благодаря дефектам на поверхности и в объеме, частицы размерами 3-5 nm обладают нескомпенсированным магнитным моментом значением $\sim 150-300 \mu_{B}[6,14-16,26,28-31]$. Ферригидрит также является составной частью биологического объекта ферритина, присутствующего в живых организмах. В „чистом виде“ ферригидрит может быть получен как химическим методом, так и путем извлечения из про- дуктов жизнедеятельности бактерий („биогенный ферригидрит“), культивированых в определенных условиях. В данной работе исследования проведены на образцах ферригидрита обоих типов.

\section{2. Эксперимент}

2.1. Получение и характеризация образцов ферригидрита. Методика получения биогенного ферригидрита, образующегося в результате жизнедеятельности бактерий Klebsiella oxytoca, подробно описана в работах [31,32]. Химический ферригидрит был получен путем медленного добавления раствора щелочи $\mathrm{NaOH}(1 \mathrm{M})$ к раствору хлорида железа $\mathrm{FeCl}_{3}$ $(0.02 \mathrm{M})$ с постоянным перемешиванием при комнатной температуре до достижения нейтрального значения $p H$ [33]. Полученные образцы проходили отжиг при $170^{\circ} \mathrm{C}$ в воздушной атмосфере в течение $24 \mathrm{~h}$. Далее по тексту эти образцы обозначены как Bio-FH и Chem-FH.

Анализ мессбауэровских спектров, полученных на спектрометре MC-1104Em (источник ${ }^{57} \mathrm{Co}(\mathrm{Cr})$ ), показал, что для полученных образцов наблюдается хорошее согласие с предыдущими данными [31-33], а проведенный отжиг не привел к появлению посторонних фаз оксида железа. По данным просвечивающей электронной микроскопии средний размер частиц $<d>$ для образцов Bio-FH и Chem-FH составил $\sim 4 \mathrm{~nm}$ и $\sim 5 \mathrm{~nm}$ соответственно.

Статические магнитные измерения намагниченности (температурные зависимости $M(T)$ ) проведены на SQUID-магнитометре [34].

2.2. Измерение петель динамического магнитного гистерезиса. Петли ДМГ измерялись при помощи индукционного магнитометра в импульсных магнитных полях, получаемых стандартным методом разрядки батареи конденсаторов через соленоид. Возможность измерения петель гистерезиса обеспечена работой установки, генерирующей импульсные магнитные поля в периодическом режиме. При этом внешнее поле увеличивалось до значения $H_{\max }$ (до $130 \mathrm{kOe}$ в данных экспериментах), затем до отрицательного значения, несколько меньшего $\left|H_{\max }\right|$, и затем до нуля (обусловлено закрытием блоков тиристоров). Продолжительность импульсов изменялась коммутацией блоков батареи конденсаторов на различные емкости. Измерения проводились при длительности импульсов $\tau_{P}\left(\tau_{P}-\right.$ время „полупериода“, за которое внешнее поле изменяется от $H=0$ до $H_{\max }$ и затем до $H=0)$, равных 4,8 и $16 \mathrm{~ms}$, что вместе с изменением максимальной напряженности поля в импульсе $H_{\max }$ позволяет производить измерения ДМГ в широком диапазоне скоростей изменения поля $d H / d t \sim 10-80 \mathrm{MOe} / \mathrm{s}$. Измерения проводились при температуре $77 \mathrm{~K}$. Скорость изменения магнитного поля в момент перемагничивания образца определялась по значению $d H / d t$ вблизи нуля из экспериментально полученных зависимостей $H(t)$. 


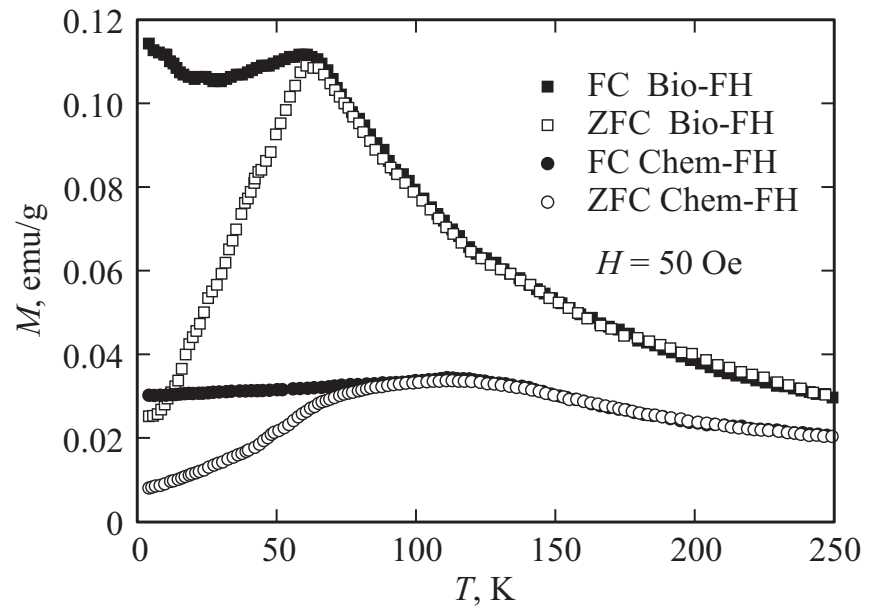

Рис. 1. Температурные зависимости намагниченности $M(T)$ исследованных образцов наноферригидрита в режимах ZFC и FC.

\section{3. Результаты и обсуждение}

На рис. 1 приведены температурные зависимости намагниченности $M(T)$ исследованных образцов, измеренные в условиях охлаждения в нулевом внешнем поле (ZFC) и внешнем поле (FC) $H=50$ Ое. Из вида этих зависимостей можно заключить, что исследуемые образцы демонстрируют характерное SP-поведение: отчетливый максимум на зависимости $M(T)_{\mathrm{ZFC}}$, расхождение зависимостей $M(T)_{\mathrm{ZFC}}$ и $M(T)_{\mathrm{FC}}$. Если определять $\mathrm{SP}$-температуру блокировки $T_{B}$ в точке максимума зависимости $M(T)_{\mathrm{ZFC}}$, то значения $T_{B}$ составляют 61 и $111 \mathrm{~K}$ для образцов Bio-FH и Chem-FH соответственно. При $T \leq T_{B}$ наблюдается расхождение зависимостей $M(T)_{\mathrm{ZFC}}$ и $M(T)_{\mathrm{FC}}$. Величины $T_{B}$ заметно превышают таковые для исходных образцов ( $\approx 23 \mathrm{~K}$ и $44 \mathrm{~K})$, не прошедших отжиг, и как показано ранее [31,35,36], рост значений $T_{B}$ связан с увеличением размеров частиц при низкотемпературном отжиге.

При температуре $T=77 \mathrm{~K}$ магнитные моменты частиц образца Вio-FH находятся в SP-состоянии для условий квазистатических магнитных измерений. Зависимости $M(H)$ этого образца при таких измерениях полностью обратимы $\left(H_{C}=0\right)$. Значение $T_{B}$ образца Chem-FH несколько превышает температуру $77 \mathrm{~K}$. Однако, по нашим данным, в квазистатических магнитных измерениях (до $60 \mathrm{kOe}$ ) при этой температуре значение $H_{C}$ не превышает $10^{2} \mathrm{Oe}$.

На рис. 2 показаны петли ДМГ для исследованных образцов. Для использованного масштаба по оси абсцисс $( \pm 155 \mathrm{kOe})$ данные $M(H)$ для всех значений максимально приложенного поля $H_{\max }$ и длительности импульса $\tau_{P}$ лежат внутри толщины линий. Вставки на рис. 2 иллюстрируют поведение петель ДМГ вблизи начала координат. Из этих данных видно, что зависимости $M(H)$ характеризуются наличием коэрцитивной силы $H_{C}$. Значение $H_{C}$ для петель ДМГ будем определять как модуль абсциссы точки пересечения зависимости $M(H)$ в осью $H$ в области $H<0)$. Значение $H_{C}$ зависит от длительности импульса и от величины $H_{\max }$. Расширение диапазона существования гистерезиса для импульсного перемагничивания достаточно легко понять, рассмотрев экспериментальные условия с помощью выражения (1). Обычно, если речь идет о температурных измерениях магнитной восприимчивости для квазистатических измерений, значение $\tau_{m}$ берут $10^{1}-10^{2} \mathrm{~s}[1]$, а если считать, что $\tau_{m}$ - время измерения петли гистерезиса, то при типичном значении $d H / d t \sim 10^{1}-10^{2}$ Ое значение $\tau_{m}$ будет составлять $\sim 10^{4} \mathrm{~s}$. Для случая переменных полей частоты $\omega$ очевидно, что $\tau_{m}=2 \pi / \omega$. Если применить такой подход для импульсных измерений, для которых $\omega=\pi / \tau_{P}$ (см. п.п. 2.2), то $\tau_{m}=2 \tau_{P}$. Тогда для наименьшей длительности импульса $\tau_{P}=4 \mathrm{~ms}$ получим (при $\left.\tau_{0} \sim 10^{-10}-10^{-11} \mathrm{~s}\right)$, что значение $T_{B}$ увеличится примерно в $1.5-1.7$ раз по сравнению с квазистационарными
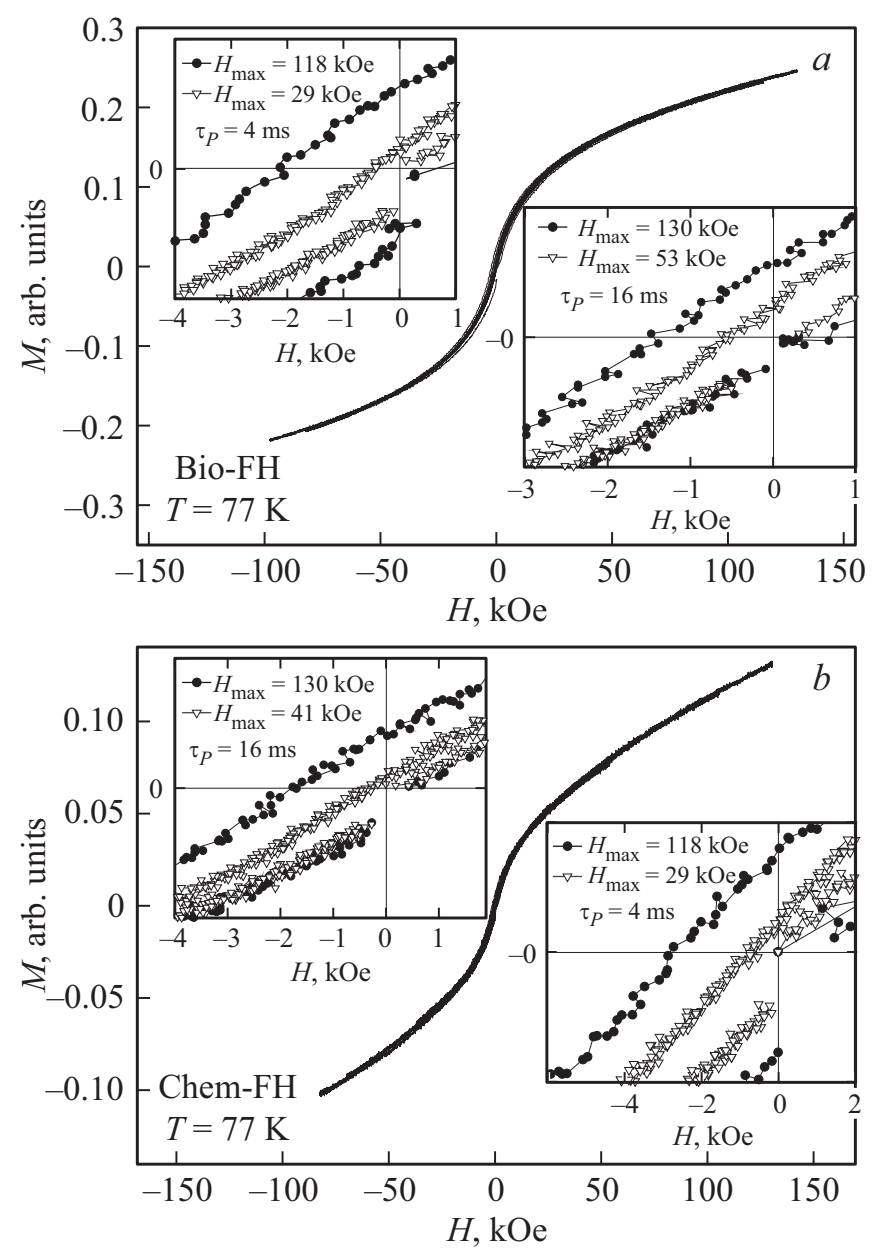

Рис. 2. Зависимости $M(H)$ при импульсном перемагничивании биогенного $(a)$ и химического $(b)$ наноферригидрита при $T=77 \mathrm{~K}$. На вставках показан ход зависимостей $M(H)$ в окрестности начала координат при различных максимальных полях $H_{\max }$ и длительностях импульса $\tau_{P}$. Отметим, что при импульсном перемагничивании внешнее поле $H$ изменяется по циклу: $0 \rightarrow H_{\max } \rightarrow-H_{\max } \rightarrow 0$. 

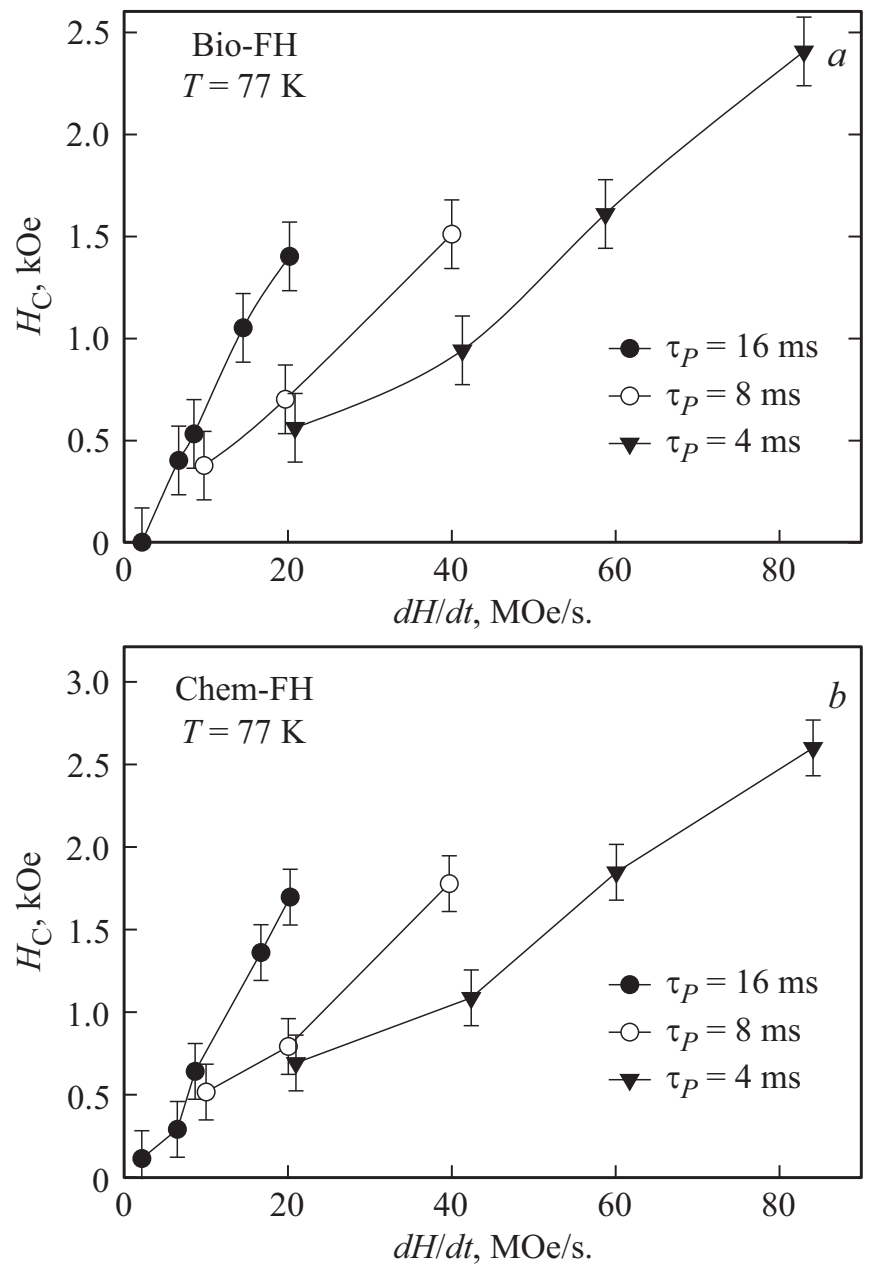

Рис. 3. Зависимости коэрцитивной силы $H_{C}$ при импульсном перемагничивании как функции скорости изменения поля $d H / d t$ для образцов биогенного $(a)$ и химического $(b)$ наноферригидрита при $T=77 \mathrm{~K}$. Данные сгруппированы (символами и соединительными линиями) по длительностям импульca $\tau_{P}$.

условиями. Это и приводит к тому, что „эффективная“ температура блокировки увеличивается, и температура измерения $77 \mathrm{~K}$ входит в диапазон, в котором магнитные моменты частиц заблокированы. В принципе, наблюдаемый рост коэрцитивной силы при увеличении частоты переменного поля $\left(\omega=\pi / \tau_{P}\right)$ не противоречит теоретическим результатам, полученным для AF-наночастиц в работе [24]. Однако на данном этапе исследований сравнение с теорией затруднительно.

На рис. 3 приведены зависимости $H_{C}$ от скорости изменения поля $d H / d t$ для исследованных образцов. Данные на рис. 3 сгруппированы (символами и соединяющими линиями) по длительностям импульса $\tau_{P}$. Видно, что, несмотря на общую тенденцию роста $H_{C}$ при увеличении значения $d H / d t$, выраженной зависимости $H_{C}(d H / d t)$ не наблюдается. И такое поведение отличается от ранее полученных данных для наночастиц $\mathcal{\varepsilon}-\mathrm{Fe}_{2} \mathrm{O}_{3}$ [27], которые применительно к процессам на- магничивания можно рассматривать как однодоменные ферро- или ферримагнитные наночастицы [37,38]. Исследуемые образцы ферригидрита проявляют AF-упорядочение, и, видимо, для такого сорта частиц присутствует дополнительный фактор, определяющий коэрцитивную силу в измерениях ДМГ. По нашему мнению, этим фактором может быть величина максимально приложенного поля. Действительно, согласно результатам исследований наноферригидрита в квазистационарных магнитных полях, эти объекты характеризуются высокими значениями полей необратимого поведения намагниченности $H_{R}[9,13]$, которые могут достигать сотен килоэрстед при низких температурах. И в результате значение $H_{C}$ зависит от величины $H_{\max }$.

С учетом вышесказанного, полученные в работе данные по коэрцитивной силе построены как функция от максимального приложенного поля $H_{\max }-$ см. рис. 4. Также как и на предыдущем рисунке, данные сгруппированы (символами и соединяющими линиями) по
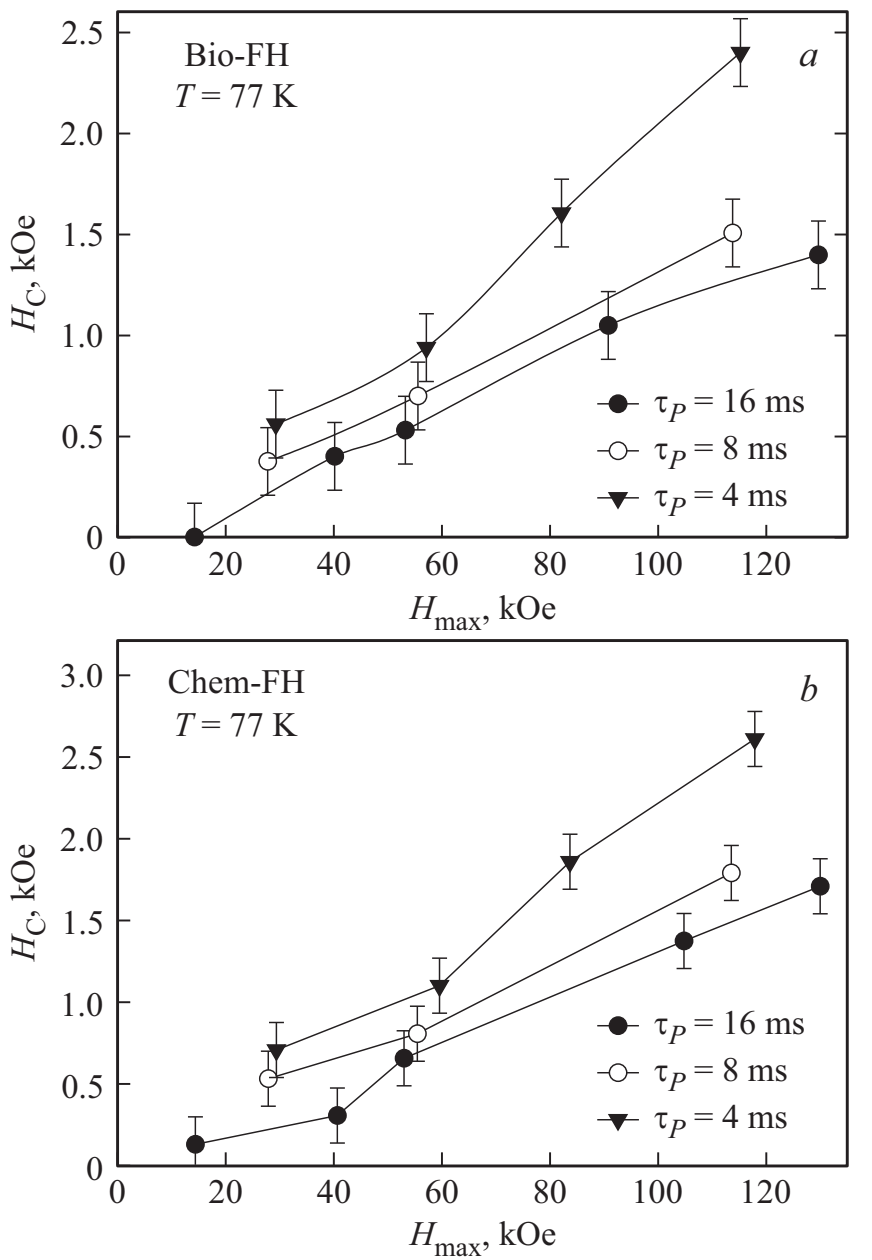

Рис. 4. Зависимости коэрцитивной силы $H_{C}$ при импульсном перемагничивании как функции от максимально приложенного поля $H_{\max }$ для образцов биогенного $(a)$ и химического $(b)$ наноферригидрита при $T=77 \mathrm{~K}$. Данные сгруппированы (символами и соединительными линиями) по длительностям импульса $\tau_{P}$. 
длительностям импульса $\tau_{P}$. Видно, что зависимости $H_{C}\left(H_{\max }\right)$ при $\tau_{P}=$ const - возрастающие функции, причем отчетливо заметно и влияние длительности импульса на величину $H_{C}$. Как было обнаружено в работax $[9,12,13]$, при квазистатических измерениях зависимости $H_{C}\left(H_{\max }\right)$ для наноферригидрита имеют $S$-образный вид. В принципе, несмотря на разброс данных рис. 4 , можно заключить, что зависимости $H_{C}\left(H_{\max }\right)$ в области полей $H_{\max }>80 \mathrm{kOe}$ характеризуются отрицательной кривизной, то есть, проявляют тенденцию к насыщению, что качественно согласуется с результатами $[9,12,13]$. Следовательно, такой параметр, как максимальное приложенное поле, является фактором, во многом определяющим поведение ДМГ наноферригидрита.

С другой стороны, если для данных рис. 4 „зафиксировать" значение $H_{\max }$ и рассмотреть зависимости $H_{C}\left(\tau_{P}\right)$ („,срез“ при условии $H_{\max } \approx$ const), то очевидно, что коэрцитивная сила возрастает с уменьшением длительности импульса: $H_{C}\left(\tau_{P}=4 \mathrm{~ms}\right)>H_{C}\left(\tau_{P}=8 \mathrm{~ms}\right)>$ $H_{C}\left(\tau_{P}=16 \mathrm{~ms}\right)$ при $H_{\max } \approx$ const. Такое поведение и можно считать проявлением эффекта динамического перемагничивания на величину коэрцитивной силы исследуемых образцов.

Можно приближенно указать, что в условиях динамического перемагничивания рост значения $H_{\max }$ приводит к увеличению $H_{C}$, примерно такому же по величине, как и уменьшение длительности импульса от $16 \mathrm{~ms}$ до $4 \mathrm{~ms}$. Например, из данных рис. 4 , увеличение значения $H_{\max }$ до $\sim 100 \mathrm{kOe}$ приводит к росту $H_{C}$ до $1.3-1.5 \mathrm{kOe}$ при $\tau_{P}=16 \mathrm{~ms}$ (при учете, что для квазистатических магнитных измерений $H_{C}$ не превышает $10^{2} \mathrm{Oe}$ ). А при $H_{\max } \sim 120 \mathrm{kOe} \mathrm{уменьшение} \tau_{P}$ от $16 \mathrm{~ms}$ до $4 \mathrm{~ms}$ приводит к дополнительному увеличению $H_{C}$ до $2.4-2.5 \mathrm{kOe}$.

\section{4. Заключение}

В данной работе экспериментально исследованы процессы динамического перемагничивания антиферромагнитных наночастиц ферригидрита двух типов (бактериального и химического). Эти исследования включали в себя импульсное перемагничивание в полях до $130 \mathrm{kOe}$ и длительностью импульса от 4 до $16 \mathrm{~ms}$. Для образцов обоих типов обнаружены следующие закономерности. В области температур, в которой в квазистационарных магнитных полях эффекты, связанные необратимым поведением кривой намагничивания, незначительны, при импульсном перемагничивании наблюдаются гистерезисные эффекты. Коэрцитивная сила зависит от обоих параметров, определяющих скорость изменения внешнего поля: длительности импульса и максимального приложенного поля. Первый фактор однозначно связан с релаксационными процессами, характерными и для ферромагнитных наночастиц: уменьшение длительности импульса эквивалентно увеличению частоты или скорости изменения поля, в результате чего коэрцитивная сила растет. Однако наблюдаемый рост коэрцитивной силы при увеличении максимального приложенного поля, вплоть до $\sim 10^{5}$ Ое, можно считать особенностью поведения не только наночастиц ферригидрита, но и наночастиц, обладающих антиферромагнитным упорядочением и нескомпенсированным магнитным моментом. По нашим данным, подобный вывод можно сделать и для наночастиц антиферромагнитного оксида никеля. Указанную особенность необходимо принимать во внимание при построении теории динамического магнитного гистерезиса антиферромагнитных наночастиц. Отметим также, что существенное расширение диапазона температур существования магнитного гистерезиса в условиях импульсных полей может быть основой для более широкого применения материалов на основе антиферромагнитных наночастиц.

Авторы благодарят Ю.В. Князева и О.А. Баюкова за мессбауэровские исследования, М.Н. Волочаева за просвечивающую электронную микроскопию образцов. Исследования проводились на просвечивающем электронном микроскопе Hitachi HT7700 Центра коллективного пользования Красноярского научного центра CO PAH.

\section{Список литературы}

[1] S. Mørup, D.E. Madsen, C. Fradsen, C.R.H. Bahl, M.F. Hansen. J. Phys.: Condens. Matter 19, 213202 (2007).

[2] Ю.Л. Райхер, В.И. Степанов, ЖЭТФ 134, 514 (2008).

[3] Yu.L. Raikher V.I. Stepanov. J. Phys.: Condens. Matter. 20, 204120 (2008).

[4] Q.A. Pankhurst, N.T.K. Thanh, S.K. Jones, J. Dobson. J. Phys. D 42, 224001 (2009).

[5] K. Dobretsov, S. Stolyar, A. Lopatin. Acta Otorhinolaryngol. Ital. 35, 2, 97 (2015)

[6] S.A. Makhlouf, F.T. Parker, F.E. Spada, A.E. Berkowitz, J. Appl. Phys. 81, 8, 5561 (1997).

[7] S.A. Makhlouf, H. Al-Attar, R.H. Kodama. Solid State Commun. 145, 1 (2008).

[8] C. Diaz-Guerra, M. Vila, J. Piqueras. Appl. Phys. Lett. 96, 193105 (2010).

[9] N. J. O. Silva, V. S. Amaral, A. Urtizberea, R. Bustamante, A. Milláan, F. Palacio, E. Kampert, U. Zeitler, S. de Brion, O. Iglesias, A. Labarta. Phys. Rev. B 84, 104427 (2011).

[10] J.F.K. Cooper, A. Ionescu, R.M. Langford, K.R.A. Ziebeck, C.H.W. Barnes, R. Gruar, C. Tighe, J. A. Darr, N.T.K. Thanh, B. Ouladdiaf. J. Appl. Phys. 114, 083906 (2013).

[11] A.E. Bianchi, S.J. Stewart, R.D. Zysler, G. Punte. J. Appl. Phys. 112, 083904 (2012).

[12] Д.А. Балаев, А.А. Красиков, А.А. Дубровский, С.В. Семенов, С.И. Попков, С.В. Столяр, Р.С. Исхаков, В.П. Ладыгина, Р.Н. Ярославцев. ФТТ 58, 2, 280 (2016).

[13] D.A. Balaev, A.A. Krasikov, A.A. Dubrovskiy, S.I. Popkov, S.V. Stolyar, R.S. Iskhakov, V.P. Ladygina, R.N. Yaroslavtsev. J. Appl. Phys. 120, 183903 (2016).

[14] N.J.O. Silva, V.S. Amaral, L.D. Carlos. Phys. Rev. B 71, 184408 (2005).

[15] S.A. Makhlouf, F.T. Parker, A.E. Berkowitz. Phys. Rev. B 55, R14717 (1997). 
[16] C. Gilles, P. Bonville, H. Rakoto, J.M. Broto, K.K.W. Wong, S. Mann. J. Magn. Magn. Mater. 241, 430 (2002).

[17] N.J.O. Silva, A. Millan, F. Palacio, E. Kampert, U. Zeitler, V.S. Amaral. Phys. Rev. B 79, 104405 (2009).

[18] Ю.Л. Райхер, В.И. Степанов, С.В. Столяр, В.П. Ладыгина, Д.А. Балаев, Л.А. Ищенко, М. Балашов. ФТТ 52, 277 (2010).

[19] Ch. Rani, S.D. Tiwari. Physica B 513, 58 (2017).

[20] Д.А. Балаев, С.И. Попков, А.А. Красиков, А.Д. Балаев, А.А. Дубровский, С.В. Столяр, Р.Н. Ярославцев, В.П. Ладыгина, Р.С. Исхаков. ФТТ 59, 10, 1920 (2017).

[21] Д.А. Балаев, А.А. Дубровский, А.А. Красиков, С.И. Попков, А.Д. Балаев, К.А. Шайхутдинов, В.Л. Кириллов, О.Н. Мартьянов. ФТТ 59, 8, 1524 (2017).

[22] I.S. Poperechny, Yu.L. Raikher, V.I. Stepanov. Phys. Rev. B 82, 174423 (2010).

[23] I.S. Poperechny Yu.L. Raikher. Physica B 435, 58 (2014).

[24] Yu.P. Kalmykov, B. Ouari, S.V. Titov. J. Appl. Phys. 120, 053901 (2016).

[25] R.H. Kodama, A.E. Berkowitz. Phys. Rev. B 59, 6321 (1999).

[26] Д.А. Балаев, А.А. Красиков, А.А. Дубровский, С.В. Семёнов, О.А. Баюков, С.В. Столяр, Р.С. Исхаков, В.П.Ладыгина, Л.А. Ищенко. ЖЭТФ 146, 546 (2014).

[27] D.A. Balaev, I.S. Poperechny, A.A. Krasikov, K.A. Shaikhutdinov, A.A. Dubrovskiy, S.I. Popkov, A.D. Balaev, S.S. Yakushkin, G.A. Bukhtiyarova, O.N. Martyanov, Yu.L. Raikher. J. Appl. Phys. 117, 063908 (2015).

[28] J.G.E. Harris, J.E. Grimaldi, D.D. Awschalom, A. Chiolero, D. Loss. Phys. Rev. B 60, 3453 (1999).

[29] Chandni Rani, S.D. Tiwari. J. Magn. Magn. Mater. 385, 272 (2015).

[30] M.S. Seehra, V.S. Babu, A. Manivannan, J.W. Lynn. Phys. Rev. B 61, 3513 (2000).

[31] D.A. Balaev, A.A. Krasikov, A.A. Dubrovskiy, S.I. Popkov, S.V. Stolyar, O.A. Bayukov, R.S. Iskhakov, V.P. Ladygina, R.N. Yaroslavtsev. J. Magn. Magn. Mater. 410, 71 (2016).

[32] С.В. Столяр, О.А. Баюков, Ю.Л. Гуревич, В.П. Ладыгина, Р.С. Исхаков, П.П. Пустошилов. Неорган. материалы 43, 6, 725 (2007).

[33] С.В. Столяр, Р.Н. Ярославцев, Р.С. Исхаков, О.А. Баюков, Д.А. Балаев, А.А. Дубровский, А.А. Красиков, В.П. Ладыгина, А.М. Воротынов, М.Н. Волочаев. ФТТ 59, 3, 538 (2017).

[34] Д.А. Великанов. Вестн. СибГАУ 2, 48, 176 (2013).

[35] Д.А. Балаев, А.А. Красиков, С.В. Столяр, Р.С. Исхаков, В.П. Ладыгина, Р.Н. Ярославцев, О.А. Баюков, А.М. Воротынов, М.Н. Волочаев, А.А. Дубровский. ФТТ 58, 9, 1724 (2016).

[36] S.V. Stolyar, D.A. Balaev, A.A. Krasikov, A.A. Dubrovskiy, R.N. Yaroslavtsev, O.A. Bayukov, M.N. Volochaev, R.S. Iskhakov. J. Supercond. Nov. Magn. (2017). https://doi.org/10.1007/s10948-017-4263-6

[37] M. Gich, A. Roig, C. Frontera, E.Molins, J. Sort, M. Popovici, G. Chouteau, D. Martın Y. Marero, J. Nogués. J. Appl. Phys. 98, 4, 044307 (2005).

[38] A.A. Dubrovskiy, D.A. Balaev, K.A. Shaykhutdinov, O.A. Bayukov, O.N. Pletnev, S.S. Yakushkin, G.M. Bukhtiyarova, O.N. Martyanov. J. Appl. Phys. 118, 213901 (2015).

Редактор К.В. Емцев 